\title{
Vacuum sponge therapy using the pull-through technique via a percutaneous endoscopic gastros- tomy to treat iatrogenic duodenal perforation
}
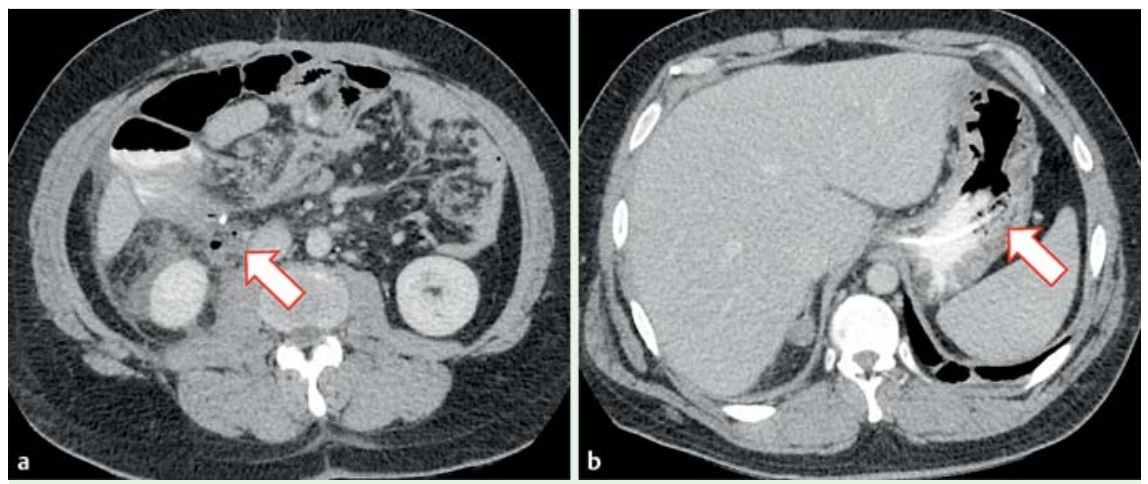

Fig. 1 a Duodenal perforation with free intra-abdominal fluid and air (arrow) in a patient undergoing argon plasma coagulation treatment for recurrent adenocarcinoma. $\mathbf{b}$ The inserted sponge (arrow) had dislocated to the stomach at 24 hours after endoscopic placement in the duodenum in order to treat the perforation shown in $\mathbf{a}$.
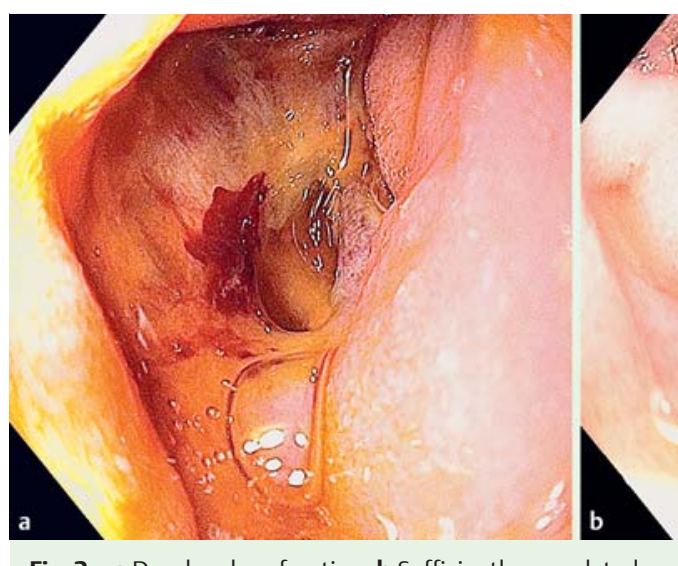

Fig. 2 a Duodenal perforation. b Sufficiently granulated cavity at 20 days after sponge treatment.

In 2011, a 37-year old patient with a history of familial adenomatous polyposis underwent a subtotal colectomy and resection of the proximal jejunum and distal duodenum with side-to-side duodenojejunostomy. The procedure was performed for adenocarcinoma of the duodenum (pT3 pNO LO V0 RO G2). In November of 2013, an endoscopically irresectable recurrent adenoma of the anastomosis was seen. Surgical resection was also impossible because of desmoids of the mesentery. Therefore, it was decided intraoperatively to resect the adenoma endoscopically in piecemeal fashion. Histology again showed adenocarcinoma (pT1 R2), and the residual carcinoma was treated at intervals of 6 months with argon plasma coagulation (APC). In June of 2015, the patient developed fever and abdominal pain 24 hours after the last APC therapy.

Computed tomography showed free fluid and air adjacent to the ablation site (৫ Fig. 1a). Endoscopy confirmed a duodenal perforation ( $\bullet$ Fig. 2 a). Because of a lack of surgical options, an Eso-Sponge (B. Braun Melsungen AG, Melsungen, Germany) was placed close to the perforation. However, post-interventional computed tomography showed that the Eso-Sponge had dislocated to the stomach ( $\bullet$ Fig. 1 b). The risk for persistent dislocation was minimized as follows: First, with a Pexact Device II (Fresenius Kabi AG, Bad Homburg, Germany), the anterior gastric wall was sutured (four polydioxanone [PDS] sutures) before a conventional 20-Fr per- cutaneous endoscopic gastrostomy (PEG) catheter (Fresenius Kabi AG) was inserted using the pull-through technique. This procedure minimizes the risk for peritonitis during frequent manipulations. A thread was introduced through the PEG into the stomach and drawn out orally with a grasping forceps. The thread was connected to the end of the Eso-Sponge tube. Second, the Eso-Sponge device was introduced into the stomach using the pull-through technique, and the EsoSponge tube was diverted through the PEG. Third, intraluminally the Eso-Sponge was positioned endoscopically close to the duodenal perforation ( Fig.3a, - Fig.3b). A negative pressure was applied (30 mmHg).

The Eso-Sponge was changed three times (at 4- to 6-day intervals) as follows: A gastric tube was connected to the external end of the Eso-Sponge tube. The EsoSponge was grasped in the duodenum, drawn out orally, and cut off. The end of a new Eso-Sponge tube was connected to the end of the transoral tube. Finally, the sponge was drawn into the stomach by the pull-through technique under endoscopic view, as described previously, and then repositioned in the duodenum.

The patient received total parenteral nutrition and antibiotic treatment (cefuroxime/metronidazole) for 10 days. Because of various risk factors (obesity, sleep apnea, lockjaw), all procedures were performed with the patient under general anesthesia. No further sponge dislocation occurred. At 20 days after the initiation of treatment, the cavity appeared closed ( $\bullet$ Fig. 2 b), and the patient was started on solid food. The PEG was removed 7 days later.

Endoscopic vacuum therapy has been established as an effective treatment for perforations [1,2]; however, it must be frequently modified in the upper gastrointestinal tract [3-5], and clinical experience is still limited. As shown in this case, intraluminal vacuum therapy is a feasible treatment option for a duodenal perforation. Because of the long distance and several angles bridged by the sponge tube and adherence to the endoscope during retrieval of the device, there is a high risk for dislocation of the sponge when it is placed in the duodenum intraluminally and drawn out nasally.

Use of the pull-through technique via PEG for sponge placement and necessary changes during treatment reduces the intraluminal distance of the Eso-Sponge 

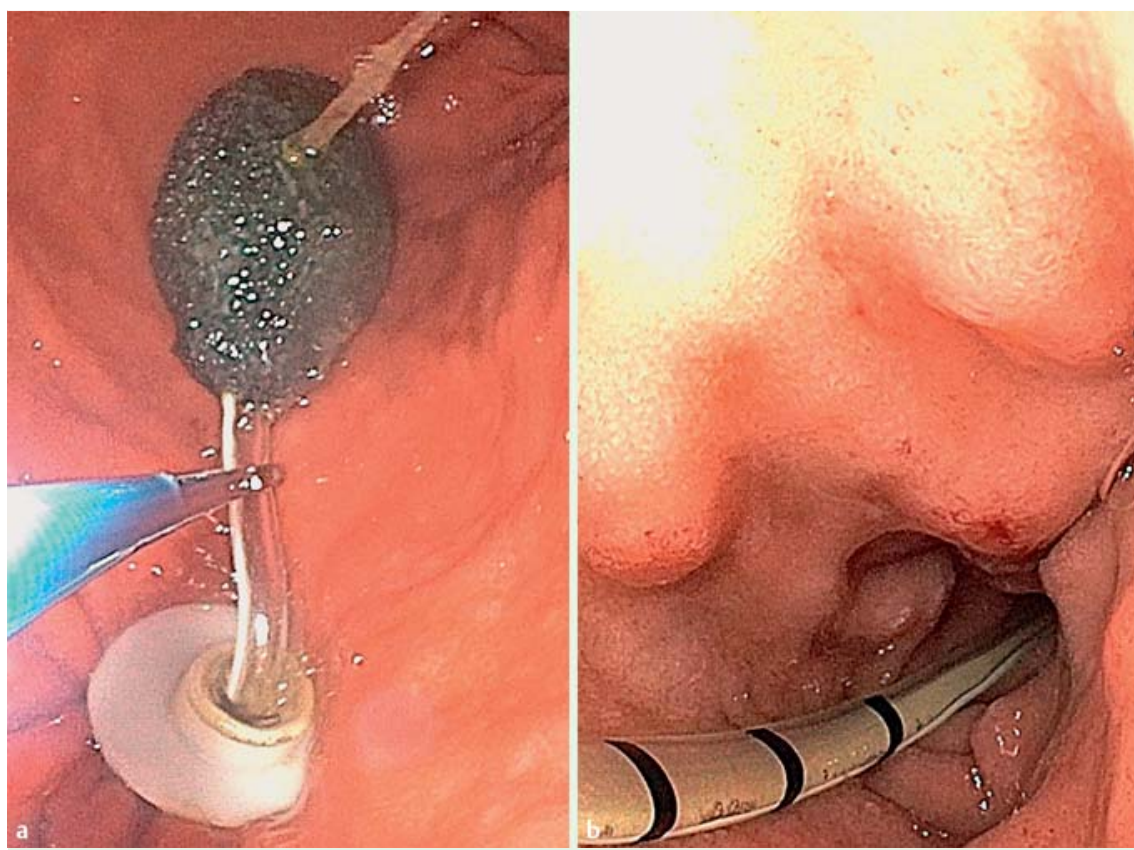

Fig. 3 a The Eso-sponge tube passes through the percutaneous endoscopic gastrostomy. $\mathbf{b}$ Endoscopic placement of the sponge in the duodenum with a forceps.

tube. Thus, the described method represents an easy way to prevent dislocation and so increase the chance of successful treatment.

Endoscopy_UCTN_Code_CPL_1AH_2AD

\section{Competing interests: None}

Torben Glatz ${ }^{1}$, Andreas Fischer ${ }^{2}$, Jens Hoeppner ${ }^{1}$, Robert Thimme ${ }^{2}$, Christine Walker $^{2}$, Hans-Jürgen Richter-Schrag ${ }^{2}$

${ }^{1}$ Department of General and Visceral Surgery, University Hospital of Freiburg, Freiburg, Germany

${ }^{2}$ University Hospital of Freiburg, Interdisciplinary Gastrointestinal Endoscopy, Department of Internal Medicine II, Freiburg, Germany

\section{References}

1 Mennigen $R$, Senninger $N$, Laukoetter $M G$ Novel treatment options for perforations of the upper gastrointestinal tract: endoscopic vacuum therapy and over-the-scope clips. World J Gastroenterol 2014; 20: 7767-7776

2 Schorsch T, Müller C, Loske G. Endoscopic vacuum therapy of anastomotic leakage and iatrogenic perforation in the esophagus. Surg Endosc 2013; 27: 2040-2045

3 Fischer A, Richter-Schrag HJ, Hoeppner J et al. Endoscopic intracavitary pull-through vacuum treatment of an insufficient pancreaticogastrostomy. Endoscopy 2014; 46 (Suppl. 01) UCTN: E218-E219

4 Fischer A, Baier P, Hopt UT et al. Laparoendoscopic mediastinal vacuum therapy of a gastric perforation through the diaphragm. Endoscopy 2011; 43 (Suppl. 02) UCTN: E393394

5 Fähndrich M, Sandmann M. A new method for endoscopic drainage of pancreatic necrosis through a gastrostomy site using an endosponge. Endoscopy 2014; 46 (Suppl. 01) UCTN: E459

\section{Bibliography}

Dol http://dx.doi.org/

10.1055/s-0034-1393369

Endoscopy 2015; 47: E567-E568

(c) Georg Thieme Verlag KG

Stuttgart · New York

ISSN 0013-726X

\section{Corresponding author}

Hans-Jürgen Richter-Schrag, MD

Hugstetter Street 55

79106 Freiburg

\section{Germany}

Fax: 49-761-270-27750

hans-juergen.schrag@uniklinik-freiburg.de 